\title{
Mean-field model for the quadrupolar phases of $\mathrm{UPd}_{3}$
}

\author{
Manh Duc Le, ${ }^{1,2,3,{ }^{*}}$ Keith A. McEwen, ${ }^{3}$ Martin Rotter, ${ }^{4,5}$ Mathias Doerr, ${ }^{6}$ Alexander Barcza, ${ }^{5,7}$ Je-Geun Park, ${ }^{1,2}$ \\ James Brooks, ${ }^{8}$ Eric Jobiliong, ${ }^{8,9}$ and David Fort ${ }^{10}$ \\ ${ }^{1}$ Center for Correlated Electron Systems, Institute for Basic Science (IBS), Seoul 151-747, Korea \\ ${ }^{2}$ Department of Physics and Astronomy, Seoul National University, Seoul 151-747, Korea \\ ${ }^{3}$ Department of Physics and Astronomy, and London Centre for Nanotechnology, University College London, \\ Gower Street, London WC1E 6BT, United Kingdom \\ ${ }^{4}$ Max Planck Institute for Chemical Physics of Solids, Nöthnitzer Str. 40, D-01187 Dresden, Germany \\ ${ }^{5}$ Institut für Physikalische Chemie, Universität Wien, Währingerstr. 42, 1090 Wien, Austria \\ ${ }^{6}$ Institut für Festkörperphysik, Technische Universität Dresden, D-01062 Dresden, Germany \\ ${ }^{7}$ Vakuumschmelze GmbH, Grünerweg 37, D-63450 Hanau, Germany \\ ${ }^{8}$ National High Magnetic Field Laboratory, 1800 Paul Dirac Dr., Tallahassee, Florida 32306, USA \\ ${ }^{9}$ Department of Industrial Engineering, Universitas Pelita Harapan Karawaci, Banten 15811, Indonesia \\ ${ }^{10}$ Department of Metallurgy and Materials, University of Birmingham, Birmingham B15 2TT, United Kingdom \\ (Received 19 March 2014; revised manuscript received 6 May 2014; published 10 June 2014)
}

\begin{abstract}
$\mathrm{UPd}_{3}$ is known to exhibit four antiferroquadrupolar ordered phases at low temperatures. We report measurements of the magnetization and magnetostriction of single crystal $\mathrm{UPd}_{3}$, along the principal symmetry directions, in fields up to $33 \mathrm{~T}$. These results have been combined with recent inelastic neutron and $\mathrm{x}$-ray resonant scattering measurements to construct a mean-field model of $\mathrm{UPd}_{3}$ including up to fourth nearest-neighbor interactions. In particular, we find that anisotropic quadrupolar interactions must be included in order to explain the lowtemperature structures derived from the scattering data.
\end{abstract}

\section{INTRODUCTION}

Low-temperature phase transitions in condensed matter systems are usually driven by the cooperative actions of the system's electronic degrees of freedom. In many cases, the exchange interactions between the spins of electrons on neighboring ions causes them to order below a characteristic transition temperature. However, in lanthanide compounds, the localized $4 f$ electrons on each ionic site may have nonspherical charge or current distributions, which may be described by a multipole expansion of their electric or magnetic fields [1]. These multipoles may interact, and in certain cases these interactions may be stronger than the spin exchange interactions, driving a phase transition to a multipolar ordered phase. There have been many examples of electric quadrupolar order observed, such as in $\mathrm{CeB}_{6}$ [2,3], $\mathrm{PrPb}_{3}$ [4], and TmTe [5], whilst higher-order electric multipoles were observed in $\mathrm{DyB}_{2} \mathrm{C}_{2}[6]$.

In contrast to the lanthanides, there have not been many cases of multipolar order observed in actinide compounds. This is due partly to the larger spatial extent of the $5 f$ wave functions, which causes them to become delocalized, and hence invalidates any description of their electric or magnetic fields about a particular point. The insulating actinide oxides $\mathrm{UO}_{2}$ and $\mathrm{NpO}_{2}$, however, have been studied extensively, and exhibit electric quadrupolar and high order magnetic multipolar order, respectively [1]. In contrast, $\mathrm{UPd}_{3}$ is one of the few metallic actinide compounds that has well-localized $5 f$ electrons, and it was one of the first compounds to be shown to exhibit quadrupolar ordering. Anomalies were observed in the heat capacity [7], thermal expansion [7],

\footnotetext{
*Corresponding author: mducle@snu.ac.kr
}

magnetic susceptibility [8], and electrical resistivity [9] of $\mathrm{UPd}_{3}$ at low temperatures, which are indicative of phase transitions. Polarized neutron diffraction measurements revealed superlattice peaks at $\mathbf{Q}=\left(\frac{1}{2} 0 l\right)$, which appear only in the non-spin-flip channel, and thus can be attributed to a structural transition [10], from hexagonal to orthorhombic symmetry.

X-ray resonant scattering (XRS) measurements [11] showed that this transition arises from the ordering of the electric quadrupole moment of the $5 f^{2}$ electrons. In addition to this phase transition at $T_{0}=7.8 \mathrm{~K}$, there are three further transitions to different antiferroquadrupolar (AFQ) ordered states at $T_{+1}=6.9 \mathrm{~K}, T_{-1}=6.7 \mathrm{~K}$, and $T_{2}=4.4 \mathrm{~K}$. Below $T_{0}$ a superlattice peak at $\left(\frac{1}{2} 0 l\right)$ where $l$ is odd is observed, whilst below $T_{-1}$ there are additional peaks at $\left(\frac{1}{2} 0 l\right)$ where $l$ is even. The $l$ odd peaks show that there is antiferroquadrupolar ordering along the $c$ direction, also denoted as an antiphase stacking of quadrupoles. The $l$ even peaks show an additional ordering of quadrupole moments in-phase along $c$.

Measurements of the order parameter using x-ray resonant scattering show that the $l$ odd order is associated mainly with $Q_{z x}$ quadrupoles [12], whilst the $l$ even order is associated with $Q_{x y}$ quadrupoles [13]. The $Q_{z x}$ ordering is accompanied by a component from the $Q_{x^{2}-y^{2}}$ quadrupoles, whilst the $Q_{x y}$ is accompanied by a $Q_{y z}$ component. The directions $x$ and $z$ are equivalent to the $a$ and $c$ crystallographic directions and $y$ is perpendicular to both. In addition, there is also an additional ordering of the $Q_{y z}$ quadrupoles in antiphase below $T_{+1}$. This sequence of phase transitions is summarized in Fig. 1.

$\mathrm{UPd}_{3}$ adopts the double hexagonal close packed $\mathrm{TiNi}_{3}$ crystal structure (space group $D_{6 h}^{4}, P 6_{3} / m m c$, no. 194) [14] with lattice parameters $a=5.76 \AA$ and $c=9.62 \AA$. This means that the nearest-neighbor U-U distance, $4.1 \AA$, is larger 


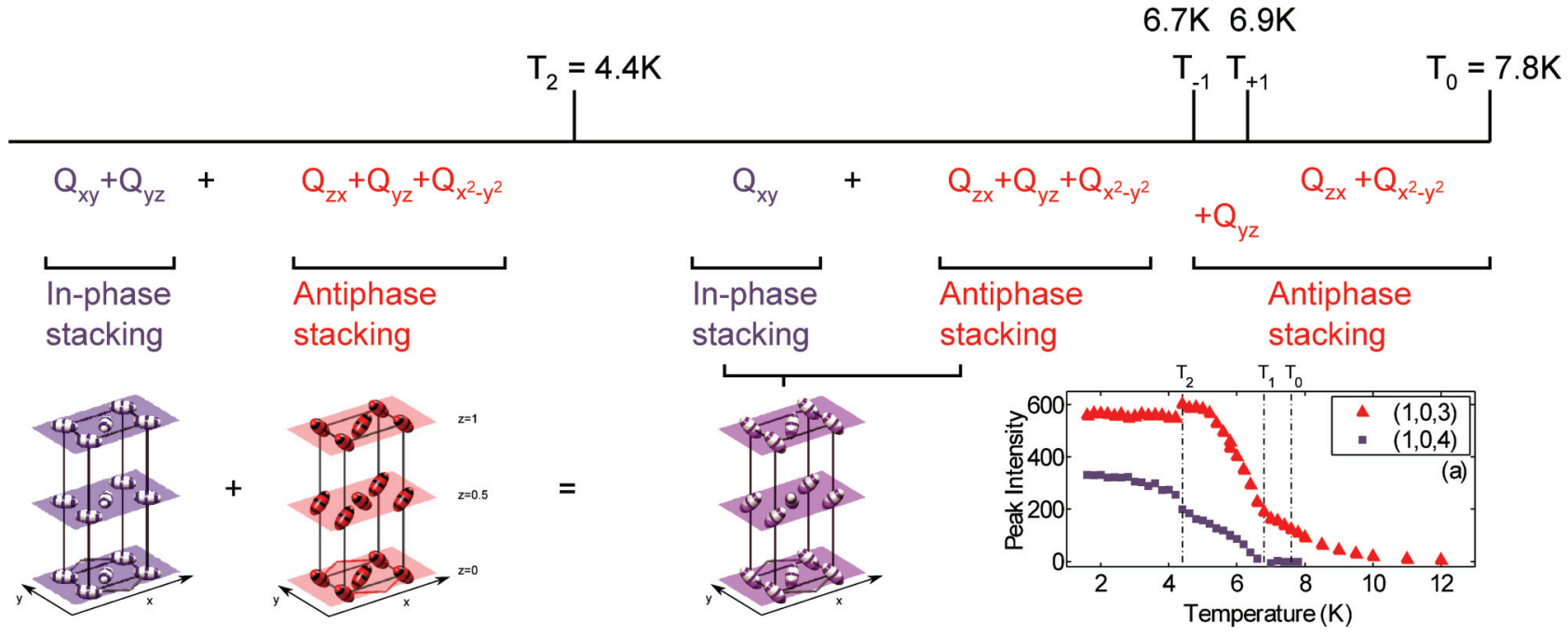

FIG. 1. (Color online) The quadrupolar phases of $\mathrm{UPd}_{3}$. Labels below the line indicate order parameter as determined from XRS data. "In phase" indicates the quadrupole moments are aligned in parallel along the $c$ axis. This corresponds to a superlattice reflection at $\mathbf{Q}=(104)$ in orthorhombic notation, or $\left(\frac{1}{2} 04\right)$ in hexagonal notation. "Antiphase" indicates an antiparallel alignment along $c$ and corresponds to a reflection at $\mathbf{Q}=\left(\begin{array}{lll}1 & 0 & 3\end{array}\right)_{\text {orth }}$ or $\left(\begin{array}{lll}\frac{1}{2} & 0 & 3\end{array}\right)_{\text {hex }}$. (After Walker et al. $\left.[12,13]\right)$.

than the Hill limit $(\approx 3.5 \AA)[15]$ and thus the $5 f^{2}$ electrons are well localized. The $\mathrm{U}^{4+}$ ions are in the $2 a$ and $2 d$ sites, which have, respectively, $D_{3 d}(\overline{3} m)$ and $D_{3 h}(\overline{6} m 2)$ point symmetry, which we shall refer to as quasicubic and hexagonal. The XRS data show that the ordering involves primarily the ions on the quasicubic sites $[12,13]$.

Knowledge of the crystal-field (CF) interactions is essential in determining the more complex two-ion interactions. In particular, it is crucial to know the CF ground state. Moreover, the excited states and the matrix elements of the angular momentum operators, $\hat{J}_{i}$, between these and the ground state determine the intensities of excitations observed by inelastic neutron scattering and also to some extent the magnitude of the magnetization in the ordered phase, as explained by McEwen et al. [8]. Thus we shall first consider in Sec. II the $\mathrm{CF}$ level scheme deduced from inelastic neutron scattering measurements in the paramagnetic phase.

We then present high-field magnetization and magnetostriction measurements in Sec. III, from which the critical fields and magnetic phase diagrams were determined experimentally. Finally, a mean-field model of the quadrupolar ordering is constructed in Sec. IV. The order parameters of each of the quadrupolar phases (determined by resonant $\mathrm{x}$-ray scattering) and their transition temperatures, were used to constrain the two-ion quadrupolar interaction parameters for the model, whilst the dipolar exchange parameters were determined from the measured critical fields. The results of the model are then compared to the measured high-field data.

Whilst the model, which we shall present, is empirically based on the measured physical properties of $\mathrm{UPd}_{3}$ rather than directly on measured exchange constants, we note that it is the first attempt to explain comprehensively this fascinating compound with its many competing ordered phases from a microscopic point of view and hope to stimulate further $a b$ initio studies of the exchange interactions involved. The quadrupolar interactions, which drive the many phase transitions in $\mathrm{UPd}_{3}$, are difficult to measure directly, because neutrons couple only to the magnetic dipoles in the system, and the energies are far too low for inelastic $\mathrm{x}$-ray scattering to resolve. However, the wealth of physical property measurements available on $\mathrm{UPd}_{3}$ has encouraged us to try to synthesize this into a mean-field model which explains, to a large extent, these varied measurements. We hope that this may encourage the construction of models to explain the properties of similar quadrupolar (or higher multipolar) ordered compounds thus deepening the understanding of what lies behind these phenomena.

\section{CRYSTAL-FIELD INTERACTIONS}

The single-ion properties of uranium are generally found to be close to the $L S$-coupling limit [16]. So, in order to simplify the analysis, we shall ignore any mixing with higher order multiplets in determining the CF parameters. As mentioned in Sec. I, there are two inequivalent sites for the $\mathrm{U}^{4+}$ ions in the crystal structure of $\mathrm{UPd}_{3}$. The different point symmetry of these sites gives rise to different crystal fields, but it happens that both split the ninefold degenerate $J=4$ ground multiplet into three singlets and three doublets. The energies and wave functions of these levels, however, are different for the two sites.

The nature of the ground state may be deduced from single crystal susceptibility measurements and estimates of the magnetic entropy determined from heat capacity measurements. These results indicate a singlet ground state on the hexagonal sites and a doublet on the quasicubic sites [8].

The CF split energy levels were determined from previously reported inelastic neutron scattering measurements made on the time-of-flight spectrometer HET at the ISIS Facility, United Kingdom [17], and on the triple-axis-spectrometer IN8 at ILL, Grenoble [18]. We identified magnetic excitations at 4.1, 9.7, $12.3,16.8,20.4$, and $30 \mathrm{meV}$. The $16 \mathrm{meV}$ peak exhibits considerable dispersion and is assigned to the transition between the $\left|J_{z}=0\right\rangle$ singlet ground state and the $\left|J_{z}= \pm 1\right\rangle$ doublet excited state of the hexagonal site ions. Its dispersion 
was used to determine the exchange interactions between hexagonal sites [19]. The remaining peaks are assigned to the quasicubic site ions.

Henceforth we shall be concerned mainly with the quasicubic sites, as the quadrupolar ordering primarily involves the uranium ions on these sites. These sites have trigonal, $\overline{3} \mathrm{~m}$ $\left(D_{3 d}\right)$, point symmetry, so that the crystal-field Hamiltonian is

$$
\mathcal{H}_{\mathrm{cf}}=\sum_{k=2,4,6} B_{k}^{0} O_{k}^{0}+\sum_{k=4,6} B_{k}^{3} O_{k}^{3}+B_{6}^{6} O_{6}^{6},
$$

where $B_{k}^{q}$ are crystal-field parameters and $O_{k}^{q}$ are Stevens operators. The quantization $(z)$ axis is taken to be the trigonal axis, which in this case is parallel to $c$.

From the measured transition energies and with the restriction of a doublet ground state, a crystal-field fitting program [20] was used to obtain initial estimates of the CF parameters for the quasicubic sites. This program relies on the orthogonality of the spherical harmonic functions from which the $\mathrm{CF}$ operators are constructed. It allows one to find a set of CF parameters, $B_{k}^{q}$, given the energy levels and wave functions produced by the crystal field. The fitting algorithm may thus vary either the wave functions to fit a particular set of energy levels, or vice versa. In this case, however, we also face constraints on the wave functions.

From symmetry considerations, the doublet ground states have the wave functions

$$
\begin{aligned}
& \left|d_{1}\right\rangle=a|4\rangle+b|1\rangle+c|-2\rangle, \\
& \left|d_{2}\right\rangle=a|-4\rangle-b|-1\rangle+c|2\rangle,
\end{aligned}
$$

where for brevity the kets denote states $\left|J=4, J_{z}\right\rangle$. The singlet wave functions have the forms

$$
\begin{aligned}
& |s\rangle=d|3\rangle+e|0\rangle-d|-3\rangle, \\
& \left|s^{\prime}\right\rangle=\frac{1}{\sqrt{2}}(|3\rangle+|-3\rangle) .
\end{aligned}
$$

In order to ensure that the $\mathrm{T}_{0}=7.8 \mathrm{~K}$ transition is accompanied by only a very small entropy change, as deduced from the heat capacity data, the Landau theory analysis of McEwen et al. [8] requires that the matrix elements $\left\langle d_{1}\left|Q_{z x}\right| d_{2}\right\rangle=$ $\left\langle d_{2}\left|Q_{z x}\right| d_{1}\right\rangle \approx 0$, where $Q_{z x}=\frac{1}{2}\left(J_{x} J_{z}+J_{z} J_{x}\right)$. As shown in the reference, this implies that $b c \approx 0$.

In addition, we note that the basal plane susceptibilities $\chi_{x, y}$ increase with decreasing temperature through the $T_{-1}=$ 6.7 $\mathrm{K}$ and $T_{2}=4.4 \mathrm{~K}$ transitions. This may be explained if the first excited state is a singlet and there is a large $\hat{J}_{x, y}$ matrix element between it and some higher-energy state which increases the $x$ - or $y$-direction susceptibility in the ordered phases as progressively more of the singlet state is mixed in with the doublet ground state [21]. As the $\hat{J}_{x, y}$ matrix elements between singlet states are zero, this coupling must be to a higher lying doublet, $\left|d_{1,2}^{(2)}\right\rangle$. The condition that $\left\langle s\left|\hat{J}_{x, y}\right| d_{1,2}^{(2)}\right\rangle$ be large, whilst $\left\langle s\left|\hat{J}_{x, y}\right| d_{1,2}\right\rangle$ is small is thus satisfied if $e \approx 1$, $b^{(2)} \approx 1$ and $b \approx 0$.

These requirements are satisfied by the crystal-field parameters in Table I, which yield $b=0.02, b^{(2)}=0.99$ and $e=0.92$. The parameters were obtained using a simulated annealing minimization procedure whereby at each iteration, the algorithm mentioned above [20] was used to refine an
TABLE I. Crystal-field parameters in Stevens normalization in $\mathrm{meV}$. The parameters were deduced from fitting to inelastic neutron spectra and the constraints on the wave functions of the lowest three energy levels of the quasicubic sites as described in the text.

\begin{tabular}{lrcc}
\hline \hline$B_{0}^{2}$ & 0.035 & $B_{0}^{6}$ & -0.00012 \\
$B_{0}^{4}$ & -0.012 & $B_{3}^{6}$ & 0.0025 \\
$B_{3}^{4}$ & -0.027 & $B_{6}^{6}$ & 0.0068 \\
\hline \hline
\end{tabular}

initial set of parameters to fit the measured energy levels. These refined parameters are subsequently used to calculate the $b, b^{(2)}$ and $e$ matrix elements, from which the simulated annealing "energy" is obtained, and hence minimized. Figure 2 shows the resulting crystal-field energy splitting and wave functions for the $\mathrm{U}^{4+}$ ions on the quasicubic sites.

We note that these energy levels, and the crystal-field parameters from which they arise, do not show any cubic symmetry. Indeed, even a point charge calculation assuming $\mathrm{Pd}^{+}$and $\mathrm{U}^{4+}$ ions yields a crystal-field splitting quite far from the cubic expectation: in common with Buyers et al. [22], we find a singlet ground state, doublets at $\approx 3.2,8.8$, and $28.6 \mathrm{meV}$ above this, and two singlets at $\approx 5.8$ and $12.7 \mathrm{meV}$. Were the crystal-field symmetry truly cubic, the two low-lying doublets and singlets would be degenerate, as deduced by the LLW [23] parameters $W=0.408 \mathrm{meV}$ and $x=0.44$, which most closely approximate the point charge parameters. Thus the term "quasicubic" is somewhat of a misnomer and has been retained here only for continuity with the literature, while the true symmetry of the crystal field at this site is trigonal.
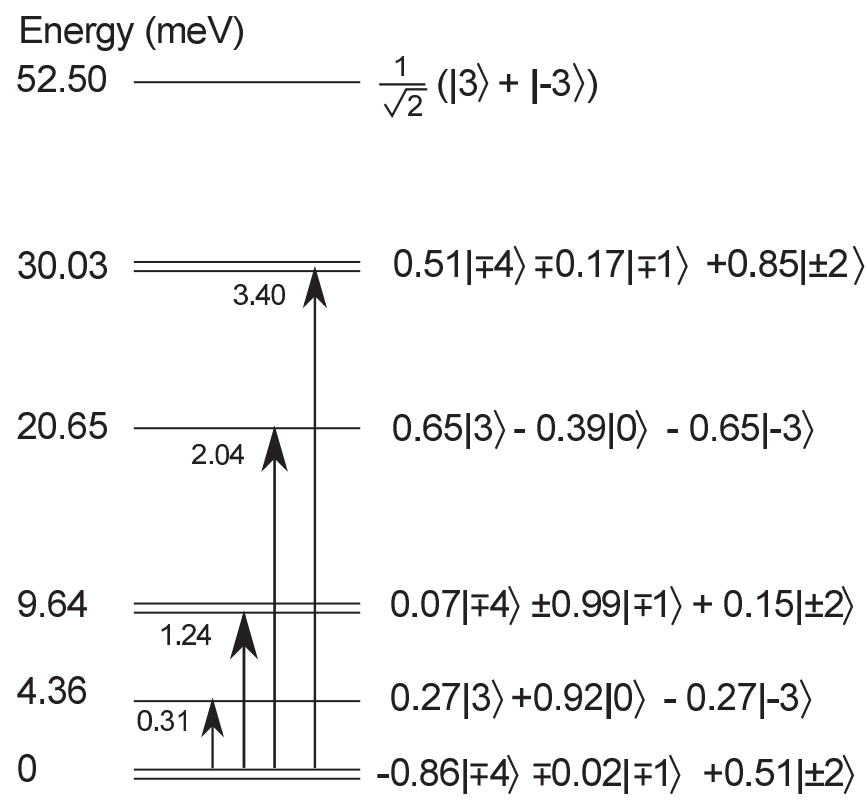

FIG. 2. $\mathrm{U}^{4+}$ quasicubic site crystal field level scheme. The crystal-field energy levels and corresponding wave functions in the paramagnetic phase expressed in the $\left|J=4, J_{z}\right\rangle$ basis. Arrows denote transitions from the ground state with non-negligible dipole matrix elements whose squared values are shown as numbers near the arrow. 


\section{HIGH-MAGNETIC FIELD MEASUREMENTS}

Single crystals were grown by the Czochralski technique at the University of Birmingham and cut with faces perpendicular to the orthogonal axes $x, y$, and $z$, where $x \| a$ and $z \| c$. These were used in magnetization and magnetostriction measurements in fields up to $14 \mathrm{~T}$ at Birkbeck College [24] by two of us (KAM and JGP). Subsequently, high-field magnetization measurements were carried out (by JGP) at the Grenoble High Magnetic Field Laboratory, and magnetostriction measurements at the National High Magnetic Field Laboratory, Tallahassee. The magnetostriction was measured using a miniature capacitance dilatometer [25] in which the single-crystal samples were mounted with either the $x, y$, or $z$ faces parallel to the capacitor plates. The dilatometer could be rotated so that the magnetic field is perpendicular to the capacitor plate allowing the transverse components of magnetostriction to be measured.

Figure 3 shows the magnetization at several different applied fields, whilst Fig. 4 collates this and other data [26] to construct the magnetic phase diagrams of $\mathrm{UPd}_{3}$. The data show the $T_{0}$ transition increasing in temperature with increasing field, which is characteristic of an antiferroquadrupolar transition. In general, three ordered phases can be identified from the data, as the phase between $T_{-1}$ and $T_{+1}$ cannot be distinguished from the magnetization data.

Figure 5 shows the forced magnetostriction data at $4.2 \mathrm{~K}$, plotted as $\Delta l / l=[l(H, T=4.2 K)-l(H=0, T=4.2 \mathrm{~K})] /$ $l(H=0, T=4.2 \mathrm{~K})$. We measured the longitudinal components of magnetostriction with the field in the $x, y$, and $z$ directions, and also the transverse components $\Delta y / y$ and $\Delta z / z$ with applied field parallel to $x$ and $\Delta x / x$ with field
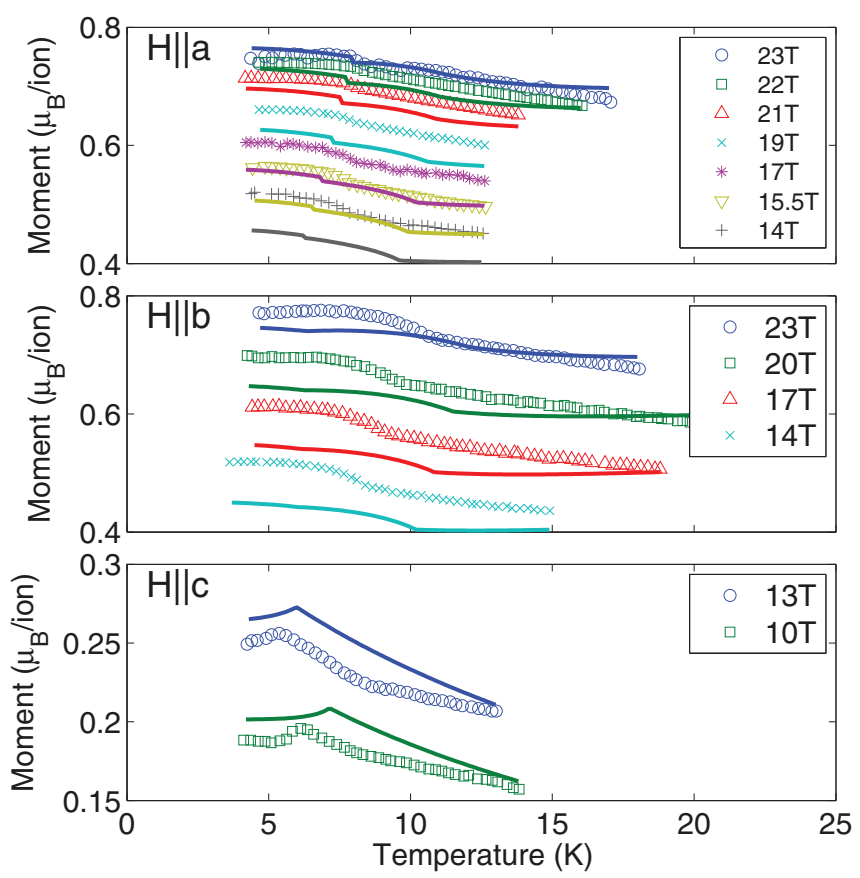

FIG. 3. (Color online) The high-field magnetization of $\mathrm{UPd}_{3}$ as a function of temperature. Solid lines are calculated from the mean-field model. The calculated values in the bottom panel (for field parallel to $c$ ) have been divided by 3 .
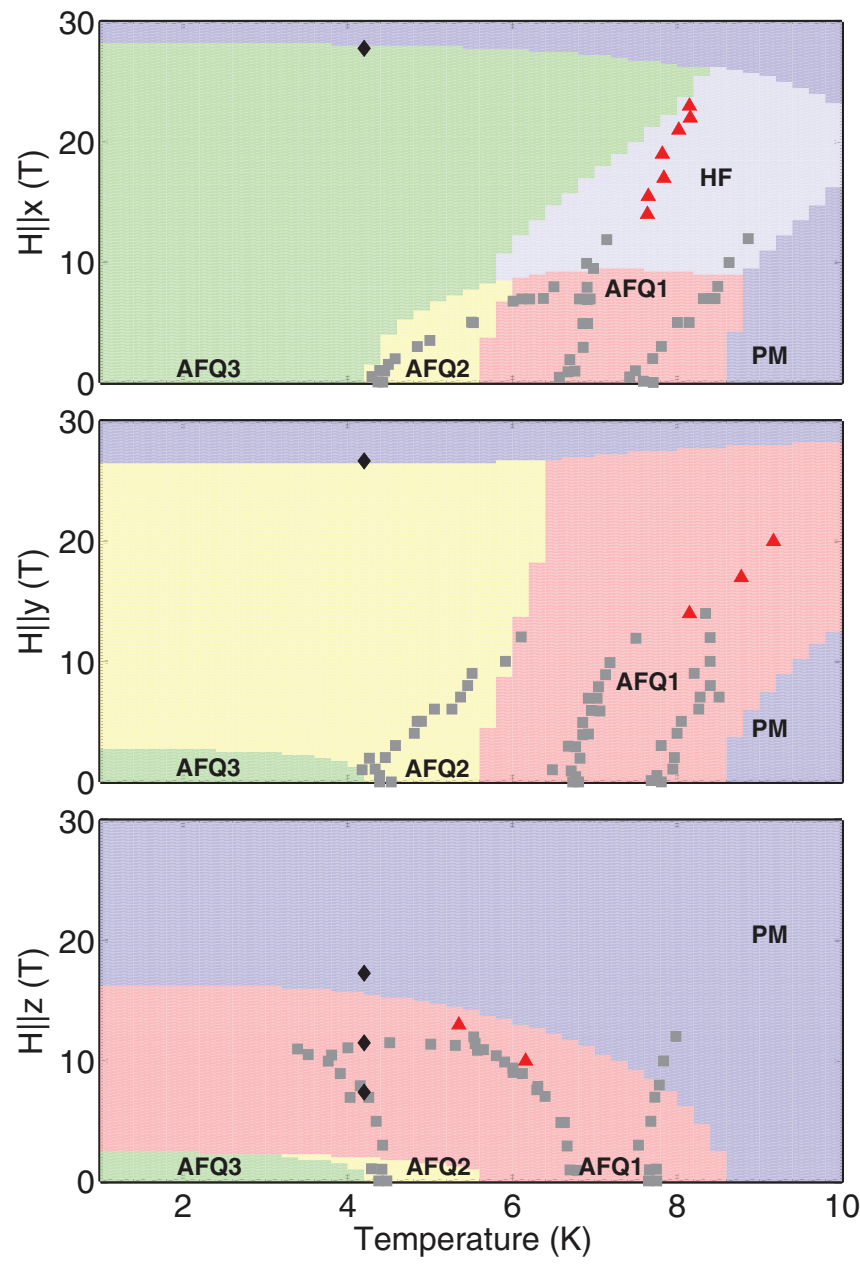

FIG. 4. (Color online) Magnetic phase diagrams of $\mathrm{UPd}_{3}$ for fields parallel to $a, b$, and $c$. Filled squares are from previous measurements by McEwen et al. [24]. Filled triangles are from high-field magnetization measurements described in this work, whilst filled diamonds are from high-field magnetostriction. The shaded background shows the MCPHASE calculated phase diagrams, with zero-field phases AFQ1-AFQ3 as described in the text. PM is paramagnetic (or ferromagnetically polarized high-field) phase, and HF is the high-field phase for $H \| a$, which has the ordering wave vector $\left(\frac{1}{2} \frac{1}{2} 0\right)$ with all quadrupolar moments ordered.

parallel to $y$. The measurements were repeated to confirm the reproducibility of the data, and subsequently, binned and averaged. In addition, the signal was corrected for artifacts due to eddy currents. The mechanical noise from the magnet cooling system and electrical noise in the leads meant that we obtained a resolution of $10^{-6}$ in $\Delta l / l$.

For $H \| x$, the magnetostriction parallel (perpendicular) to the applied field first decreases (increases) until approximately $3 \mathrm{~T}$, then increases (decreases) to about $15 \mathrm{~T}$ before decreasing (increasing) slightly. Similar, but less pronounced, behavior is also observed for $H \| y$.

Transitions at high field were observed when the field was applied in the basal plane, with a slight anisotropy between the $x$ and $y$ directions. With applied field parallel to the $x$ direction we see a steplike change in both the longitudinal and transverse magnetostriction at $28 \mathrm{~T}$, whereas for field parallel 


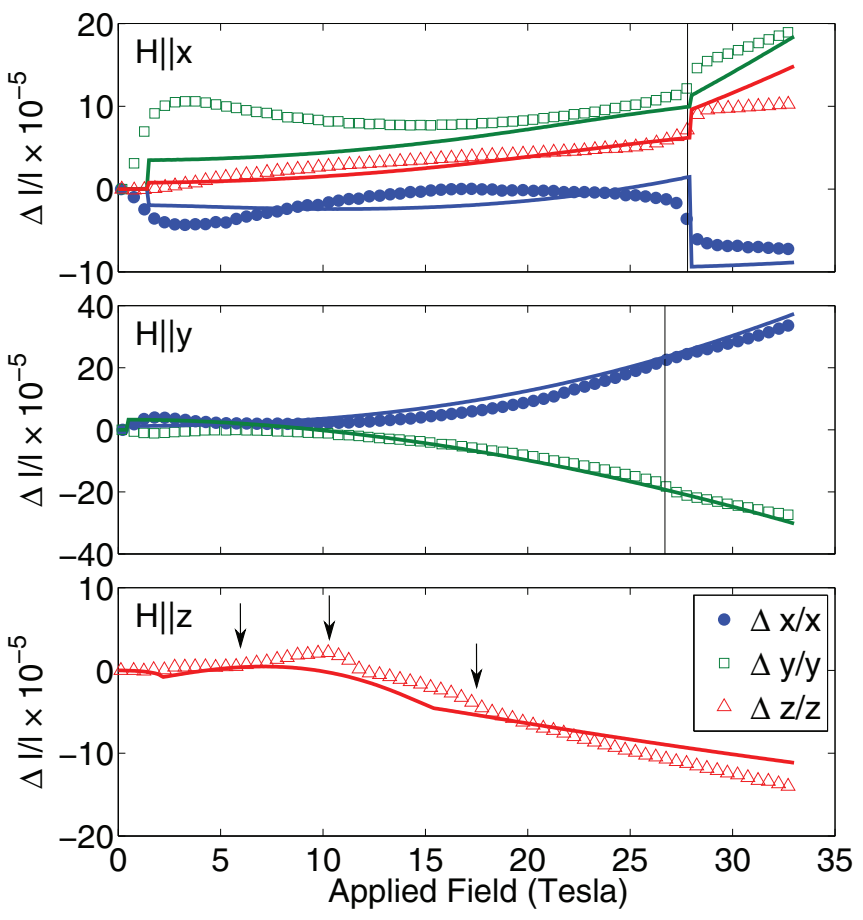

FIG. 5. (Color online) The high-field longitudinal and transverse magnetostriction of $\mathrm{UPd}_{3}$ at $4.2 \mathrm{~K}$. Solid vertical lines indicate the observed high-field transitions. Blue circles, green squares and red triangles denote the magnetostriction parallel to the $x, y$, and $z$ directions, respectively, for the indicated field directions. Solid lines are the results of the mean-field calculations. to the $y$ direction a change of slope is observed at $27 \mathrm{~T}$. For both field directions, the magnetostriction parallel to the field decreases (the crystal contracts) at the phase transition, whilst the magnetostriction perpendicular to the field increases (the crystal expands).

When the field is applied parallel to the $z$ direction, we observed no high-field transitions above $20 \mathrm{~T}$, but instead see anomalies (indicated by arrows in Fig. 5) in the longitudinal magnetostriction, at 7,11 , and $17 \mathrm{~T}$, in agreement with our magnetization data and the phase diagram of Tokiwa et al. [9].

\section{QUADRUPOLAR TWO-ION INTERACTIONS AND MEAN-FIELD MODEL FOR UPD 3}

The ordered quadrupolar structures were calculated from a mean-field model, with quadrupolar interactions between the quasicubic site ions, using the package MCPHASE $[27,28]$. In order to determine the stable ordered structure, a set of supercells and corresponding wave vectors is generated. From the wave vector, a configuration of moments (dipole, quadrupole, etc.) is generated and used as an initial configuration for a self-consistent mean-field calculation. For each solution of the mean-field iteration, the free energy is calculated. The self-consistent ordered structure with the lowest free energy is taken to be stable and used for the computation of the physical properties. In this way, the phase boundaries between the different quadrupolar ordered structures were determined in order to construct the magnetic phase diagrams of $\mathrm{UPd}_{3}$.

The Hamiltonian

$$
\begin{aligned}
\mathcal{H}= & \sum_{i}\left\{\mathcal{H}_{\mathrm{cf}}^{i}+\mathcal{H}_{Z}^{i}\right\}-\frac{1}{2}\left\{\mathcal{J}_{11}^{i j} \sum_{i j}^{i j}\left(\hat{J}_{x}^{i} \hat{J}_{x}^{j}+\hat{J}_{y}^{i} \hat{J}_{y}^{j}\right)+\mathcal{K}_{11}^{i j} \sum_{i j}^{i j}\left[\cos \left(2 \phi_{i j}\right)\left(\hat{J}_{x}^{i} \hat{J}_{x}^{j}-\hat{J}_{y}^{i} \hat{J}_{y}^{j}\right)-\sin \left(2 \phi_{i j}\right)\left(\hat{J}_{x}^{i} \hat{J}_{y}^{j}+\hat{J}_{y}^{i} \hat{J}_{x}^{j}\right)\right]\right. \\
& +\mathcal{J}_{10}^{i j} \sum_{i j}^{i j} \hat{J}_{z}^{i} \hat{J}_{z}^{j}+\mathcal{J}_{21}^{i j} \sum_{i j}^{i j}\left(\hat{Q}_{z x}^{i} \hat{Q}_{z x}^{j}+\hat{Q}_{y z}^{i} \hat{Q}_{y z}^{j}\right)+\mathcal{K}_{21}^{i j} \sum_{i j}^{i j}\left[\cos \left(2 \phi_{i j}\right)\left(\hat{Q}_{z x}^{i} \hat{Q}_{z x}^{j}-\hat{Q}_{y z}^{i} \hat{Q}_{y z}^{j}\right)\right. \\
& \left.+\sin \left(2 \phi_{i j}\right)\left(\hat{Q}_{z x}^{i} \hat{Q}_{y z}^{j}+\hat{Q}_{y z}^{i} \hat{Q}_{x z}^{j}\right)\right]+\mathcal{J}_{22}^{i j} \sum_{i j}^{i j}\left(\hat{Q}_{x^{2}-y^{2}}^{i} \hat{Q}_{x^{2}-y^{2}}^{j}+\hat{Q}_{x y}^{i} \hat{Q}_{x y}^{j}\right) \\
& \left.+\mathcal{K}_{22}^{i j} \sum_{i j}^{i j}\left[\cos \left(4 \phi_{i j}\right)\left(\hat{Q}_{x^{2}-y^{2}}^{i} \hat{Q}_{x^{2}-y^{2}}^{j}-\hat{Q}_{x y}^{i} \hat{Q}_{x y}^{j}\right)-\sin \left(4 \phi_{i j}\right)\left(\hat{Q}_{x y}^{i} \hat{Q}_{x^{2}-y^{2}}^{j}+\hat{Q}_{x^{2}-y^{2}}^{i} \hat{Q}_{x y}^{j}\right)\right]\right\}
\end{aligned}
$$

was employed, where the site indices $i$ and $j$ run over nearest- $(i j=\mathrm{nn})$ and next-nearest neighbors $(i j=\mathrm{nnn})$ within an $a b$ plane and nearest- $(i j=$ nnc) and next-nearest neighbors $(i j=\mathrm{n} 3 \mathrm{c})$ between planes, and $\phi_{i j}$ is the angle in the $a b$ plane between the projection of the vector between $i$ and $j$ in this plane and the $x(a)$ axis. $\mathcal{H}_{\mathrm{cf}}^{i}$ is the crystal-field Hamiltonian of the $i$ th quasicubic ion given in Eq. (1) and $\mathcal{H}_{Z}^{i}$ is the Zeeman Hamiltonian, $-g_{J} \mu_{B} \mathbf{J}_{i} \cdot \mathbf{H}$. The form of the two-ion exchange Hamiltonian was derived by considering a high-symmetry anisotropic interaction between each pair of neighbors in a local coordinate system defined by an $x^{\prime}$ axis along the projection of the bond in the $a b$ plane, and $z^{\prime}$ axis along $c$, and then rotating them into a global coordinate system [29,30] as described in Appendix. In this way, the full hexagonal symmetry of the interactions is satisfied. Such expressions were used to explain the properties of elemental $\operatorname{Pr}[31]$, and the dispersion of crystal-field excitations due to interactions between the hexagonal sites in $\mathrm{UPd}_{3}$ [19]. We note that the form of the local anisotropic interaction chosen is higher than that demanded by the symmetry of the midpoint between two quasicubic sites $\left(C_{2 h}\right)$, but is required in order to ensure that the dispersive crystal-field modes remain degenerate at certain high-symmetry $q$ vectors as observed in the neutron scattering data [19].

The Hamiltonian (4) suffices to describe the ordered phases of $\mathrm{UPd}_{3}$. In particular, the anisotropic exchange terms $(\propto \mathcal{K})$ are required since the first (second) order isotropic quadrupolar interactions couple the $\hat{Q}_{z x}\left(\hat{Q}_{x y}\right)$ and $\hat{Q}_{y z}\left(\hat{Q}_{x^{2}-y^{2}}\right)$ operators 
equally, but the principal order parameters measured by resonant x-ray diffraction are $\hat{Q}_{z x}$ and $\hat{Q}_{x y}$. Thus the anisotropic terms are required to favour these $\left(\hat{Q}_{z x}, \hat{Q}_{x y}\right)$ interactions over the others $\left(\hat{Q}_{y z}, \hat{Q}_{x^{2}-y^{2}}\right)$.

In order to stabilize a structure with antiphase (in-phase) stacking along $c$, we require $\mathcal{J}^{\text {nnc }}<0\left(\mathcal{J}^{\text {nnc }}>0\right)$, whilst the AFQ order in the $a b$ plane requires $\mathcal{J}^{\mathrm{nn}, \mathrm{nnn}}<0$. Thus

$$
\begin{gathered}
\mathcal{J}_{z x}^{\mathrm{nnc}}=\mathcal{J}_{21}^{\mathrm{nnc}}+\mathcal{K}_{21}^{\mathrm{nnc}} \cos 2 \phi_{\mathrm{nnc}}<0, \\
\mathcal{J}_{x y}^{\mathrm{nnc}}=\mathcal{J}_{22}^{\mathrm{nnc}}-\mathcal{K}_{22}^{\mathrm{nnc}} \cos 4 \phi_{\mathrm{nnc}}>0, \\
\left|\mathcal{J}_{z x}^{\mathrm{nnc}}\right|>\left|\mathcal{J}_{x y}^{\mathrm{nnc}}\right|, \\
\left(\mathcal{J}_{21}^{\mathrm{nn}}-\mathcal{K}_{21}^{\mathrm{nn}} \cos 4 \phi_{\mathrm{nn}}\right)<\left(\mathcal{J}_{22}^{\mathrm{nn}}+\mathcal{K}_{22}^{\mathrm{nn}} \cos 2 \phi_{\mathrm{nn}}\right)<0
\end{gathered}
$$

should be satisfied in order to yield two phases with $\hat{Q}_{z x}$ antiphase ( $\hat{Q}_{x y}$ in-phase) order along $c$ at higher (lower) temperatures, as measured. Furthermore, because the $\hat{Q}_{z x}\left(\hat{Q}_{x y}\right)$ and $\hat{Q}_{x^{2}-y^{2}}\left(\hat{Q}_{y z}\right)$ operators share the same symmetry [8], an ordering of one of these pairs will induce a secondary ordering of the other quadrupole of the pair on the same site. That is, a nonzero expectation value $\left\langle\hat{Q}_{z x}\right\rangle$ implies $\left\langle\hat{Q}_{x^{2}-y^{2}}\right\rangle \neq 0$ also (angled brackets denote the thermal expectation values $\langle\hat{O}\rangle=$ $\sum_{n}\langle n|\hat{O}| n\rangle \exp \left(\frac{-E_{n}}{k_{B} T}\right) / Z$, where the states $|n\rangle$ are eigenstates of the Hamiltonian (4) and $Z$ is the partition function). This contributes to the effective field acting on an ion and combines with the exchange interaction to reinforce (or suppress) the ordering of some particular quadrupole. The strength of this contribution is dependent on the crystal-field wave functions, and we found that for $\mathrm{UPd}_{3}$, the $\left\langle\hat{Q}_{x^{2}-y^{2}}\right\rangle$ moment induced by a $\hat{Q}_{z x}$ order is of the same order of magnitude as the primary $\left\langle\hat{Q}_{z x}\right\rangle$ moment and acts to reinforce the $\hat{Q}_{x^{2}-y^{2}}$ ordering (induced $\left\langle\hat{Q}_{x^{2}-y^{2}}\right\rangle>0$ ). This means that, unfortunately, in our model, there is always a large $\hat{Q}_{x^{2}-y^{2}}$ moment in disagreement with the measured XRS azimuthal dependence, which indicates a 10\%-15\% contribution.

The other induced moments are generally an order of magnitude weaker than their primary order parameter. They are needed, though, to account for the observation by inelastic neutron scattering of four almost dispersionless excitations below $4 \mathrm{meV}$ at $1.8 \mathrm{~K}$, which arise from transitions between the levels of the ground state doublet on the quasicubic sites, whose degeneracy is lifted by the quadrupolar order. As there are four quasicubic sites in the ordered unit cell, this implies that the splitting on each site is different, which may only occur if the magnitude of the quadrupolar moments on each site is different. In-phase ordering of the quadrupoles along $c$ (denoted $\mathrm{CS}$ for $c$ same in the following for brevity) means that the moments on sites $\mathrm{C} 1-\mathrm{C} 4$ of Fig. 6 are $\left\langle\hat{Q}^{\mathrm{C} 1}\right\rangle=\left\langle\hat{Q}^{\mathrm{C} 2}\right\rangle=-\left\langle\hat{Q}^{\mathrm{C} 3}\right\rangle=-\left\langle\hat{Q}^{\mathrm{C} 4}\right\rangle$, whilst antiphase ordering (henceforth denoted CD, $c$-different) implies that $\left\langle\hat{Q}^{\mathrm{C} 1}\right\rangle=-\left\langle\hat{Q}^{\mathrm{C} 2}\right\rangle=-\left\langle\hat{Q}^{\mathrm{C} 3}\right\rangle=\left\langle\hat{Q}^{\mathrm{C} 4}\right\rangle$. However, since $\left|\left\langle\hat{Q}_{x y}\right\rangle\right|=\left|\left\langle\hat{Q}_{x^{2}-y^{2}}\right\rangle\right|$ and $\left|\left\langle\hat{Q}_{z x}\right\rangle\right|=\left|\left\langle\hat{Q}_{y z}\right\rangle\right|$ [8], the combination of $\hat{Q}_{z x} \mathrm{CD}, \hat{Q}_{y z} \mathrm{CD}, \hat{Q}_{x y} \mathrm{CS}, \hat{Q}_{x^{2}-y^{2}} \mathrm{CS}$ ordering imposed by the Hamiltonian (4) and conditions (5) and (6) will result in $\left\langle\hat{Q}^{\mathrm{C} 1}\right\rangle=-\left\langle\hat{Q}^{\mathrm{C} 3}\right\rangle=\left\langle\hat{Q}_{x y}\right\rangle$ and $\left\langle\hat{Q}^{\mathrm{C} 2}\right\rangle=-\left\langle\hat{Q}^{\mathrm{C} 4}\right\rangle=\left\langle\hat{Q}_{z x}\right\rangle$ so that only two excitations may be expected. Only by including the induced moments, which yields $\hat{Q}_{x^{2}-y^{2}} \mathrm{CD}$ and $\hat{Q}_{y z} \mathrm{CS}$ ordering (amongst others), will the moments on each of the

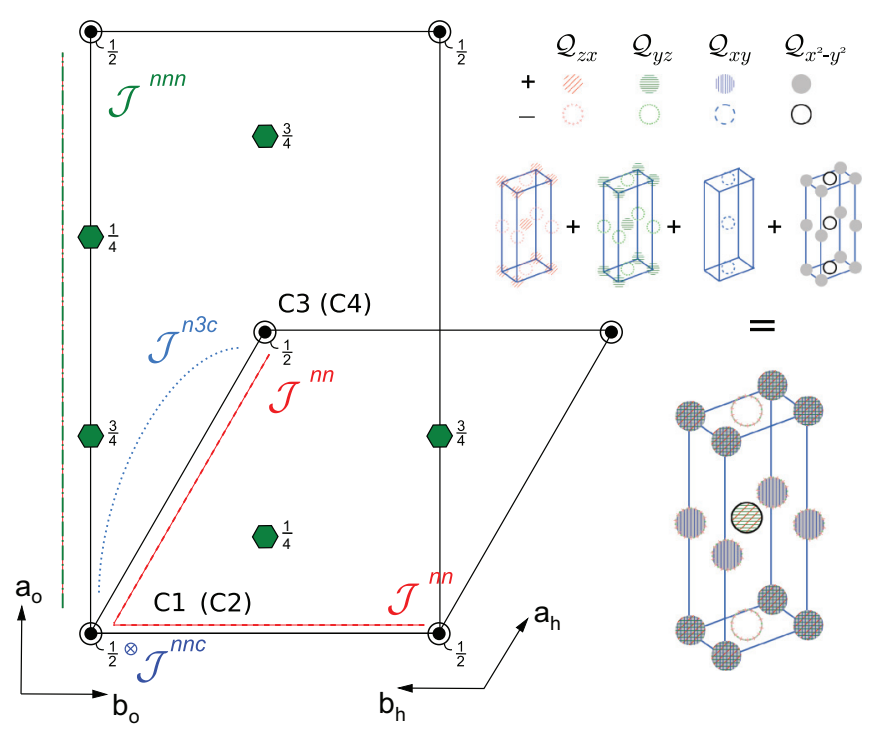

FIG. 6. (Color online) The double-hexagonal close packed structure. Only $\mathrm{U}$ atoms are shown, each of which has sixfold coordination with neighboring $\mathrm{Pd}$ atoms. Circles indicate the quasicubic, whilst hexagons the hexagonal sites. Labels in brackets apply to atoms at $z=\frac{1}{2}$. Dashed lines show the exchange interaction pathways. Figures on the right illustrate the combination of order parameters, which lead to a different quadrupolar moments on each quasicubic site in the unit cell, and thus to four transitions observed by inelastic neutron scattering at $2 \mathrm{~K}$, as explained in the text.

sites $\mathrm{C} 1-\mathrm{C} 4$, and thus the splitting of the ground state doublet, be unique. This is illustrated schematically on the right side of Fig. 6.

Finally, we calculate that for the CF scheme outlined in Sec. II, $\left\langle d_{1,2}\left|\hat{Q}_{z x}\right| s\right\rangle$ and $\left\langle d_{1,2}\left|\hat{Q}_{y z}\right| s\right\rangle$ are an order of magnitude lower than $\left\langle d_{1,2}\left|\hat{Q}_{x y}\right| s\right\rangle$ and $\left\langle d_{1,2}\left|\hat{Q}_{x^{2}-y^{2}}\right| s\right\rangle$, which means that the exchange parameters $\mathcal{J}_{21}$ should be an order of magnitude larger than $\mathcal{J}_{22}$ to give similar ordering temperatures for the first and second order quadrupoles as observed [32]. From the above considerations, we first determined the order of magnitude of exchange parameters that result in ordering temperatures below $10 \mathrm{~K}$. Subsequently, a simulated annealing and particle swarm optimization [33] search was carried out to find sets of parameters that yield at least two transitions at $\approx 4$ and $\approx 8 \mathrm{~K}$ and a splitting of the ground-state doublet at $2 \mathrm{~K}$ close to the measured values $1.28,1.68,2.20$, and $2.60 \mathrm{meV}$. Additional criteria, including the requirement that the calculated magnetization with an in-plane applied field should increase with decreasing temperature, and that there should be nonzero moments of all quadrupoles in the lowest temperature phases were then used to sort the candidate sets of parameters found by the search. We then calculated the magnetic phase diagram for the four best sets of parameters, and selected the set which have phase boundaries most similar to those measured. Finally, the parameters were refined by hand to better match the transition temperatures and fields.

We found during this procedure that unless the dipolar interactions are included, structures with ordering wave vectors $\left(\frac{1}{2} \frac{1}{2} 0\right)$ are favoured over the $\left(\frac{1}{2} 00\right)$ observed (indexed with respect to the dhcp cell). Thus small values of $\mathcal{J}_{1 m}$ and $\mathcal{K}_{1 m}$ 
TABLE II. Deduced exchange parameters in meV.

\begin{tabular}{|c|c|c|c|c|c|c|c|}
\hline $\mathcal{J}_{10}^{\text {nn }}$ & -0.017 & & & $\mathcal{J}_{10}^{\text {nnc }}$ & -0.04 & & \\
\hline $\mathcal{J}_{11}^{\text {nn }}$ & -0.01 & $\mathcal{K}_{11}^{\mathrm{nn}}$ & -0.02 & $\mathcal{J}_{11}^{\mathrm{nnc}}$ & -0.01 & $\mathcal{K}_{11}^{\mathrm{nnc}}$ & 0 \\
\hline $\mathcal{J}_{21}^{\mathrm{nn}}$ & 0.01827 & $\mathcal{K}_{21}^{\mathrm{nn}}$ & 0.00107 & $\mathcal{J}_{21}^{\mathrm{nnc}}$ & -0.06088 & $\mathcal{K}_{21}^{\mathrm{nnc}}$ & -0.00583 \\
\hline $\mathcal{J}_{21}^{\mathrm{nnn}}$ & $-1.16 \times 10^{-5}$ & $\mathcal{K}_{21}^{\mathrm{nnn}}$ & 0.00778 & $\mathcal{J}_{21}^{\mathrm{n} 3 \mathrm{c}}$ & -0.00973 & $\mathcal{K}_{21}^{\mathrm{n} 3 \mathrm{c}}$ & $-2.25 \times 10^{-4}$ \\
\hline $\mathcal{J}_{22}^{\mathrm{nn}}$ & $-8.42 \times 10^{-4}$ & $\mathcal{K}_{22}^{\mathrm{nn}}$ & $-3.65 \times 10^{-4}$ & $\mathcal{J}_{22}^{\mathrm{nnc}}$ & $-2.55 \times 10^{-5}$ & $\mathcal{K}_{22}^{\mathrm{nnc}}$ & $0.52 \times 10^{-5}$ \\
\hline $\mathcal{J}_{22}^{\mathrm{nnn}}$ & -0.00348 & $\mathcal{K}_{22}^{\mathrm{nnn}}$ & 0.00173 & $\mathcal{J}_{22}^{\mathrm{n} 3 \mathrm{c}}$ & 0.00318 & $\mathcal{K}_{22}^{\mathrm{n} 3 \mathrm{c}}$ & $2.12 \times 10^{-4}$ \\
\hline
\end{tabular}

were included, but not varied in the search procedure. They were subsequently refined by hand along with the quadrupolar interaction parameters to better fit the measured critical fields.

The final parameters are shown in Table II. At $2 \mathrm{~K}$, the full mean-field model with these parameters yields a splitting of the doublet ground state on ions C1-C4 of 0.69, 1.65, 1.82, and $2.08 \mathrm{meV}$, respectively, [at $\mathbf{Q}=\left(\frac{2}{3} \frac{2}{3} 0\right)$ ], which is somewhat lower than the experimentally measured values $(1.28,1.68$, 2.20 , and $2.60 \mathrm{meV}$ [34]). The dispersion of the levels along $[00 l], \approx 0.5 \mathrm{meV}$, is close to the measured value but the in-plane dispersion of $\approx 1 \mathrm{meV}$ is in stark contrast to the measurements, which showed the modes to be almost dispersionless.

Furthermore, the calculated order parameters differ from the XRS measurements: in the model, the dominant order parameters are $\hat{Q}_{x^{2}-y^{2}}$ and $\hat{Q}_{x y}$ rather than $\hat{Q}_{z x}$ and $\hat{Q}_{x y}$. The phase denoted AFQ1 in Fig. 4 has a large $\hat{Q}_{x^{2}-y^{2}}$ moment (ordered in antiphase along $c$ ) inducing a small $(\approx 2 \%) \hat{Q}_{z x}$ moment. In the AFQ2 phase, $\hat{Q}_{x y}$ moments become ordered, inducing some $\hat{Q}_{y z}$ quadrupoles; both these quadrupolar moments double below the transition to the AFQ3 phase. Thus although the calculated sequence of ordering agrees with experimental data, the type of quadrupolar order does not. Unfortunately, we found that it is impossible to stabilize the $\hat{Q}_{z x}$ order parameter over the $\hat{Q}_{x^{2}-y^{2}}$ order parameter at higher temperatures whilst maintaining a ground state with all quadrupolar moments ordered. This may be due to limitations in the mean-field approximation or may indicate that cooperative effects between the considered quadrupoles are important, which might be modeled, for example, by exact diagonalization or Monte Carlo methods. Alternatively, couplings not considered here, for example, between first $(q=|1|)$ and second $(q=|2|)$ order quadrupoles such as terms of the form $\hat{Q}_{z x} \hat{Q}_{x^{2}-y^{2}}$, may be required.

Another discrepancy between the model and experiments is the very low critical fields when $H \| c$ (seen at the bottom of Fig. 4) and the magnitude of the $c$ axis magnetization, which is some three times smaller than measured (Fig. 3). In principle, this can be altered by increasing the $\mathcal{J}_{10}$ exchange parameters, however, we found that raising these from the values in Table II suppresses the quadrupolar ordering completely, in favor of a dipolar order. Alternatively, the quadrupolar exchange parameters $\mathcal{J}_{2 m}$ may be altered, but an increase in the critical field necessitates also an increase in the transition temperatures.

Since the exchange interactions are likely to arise from the RKKY mechanism, one expects that it should be long ranged. Thus including interactions further than nearest neighbor may give better agreement with the data. However, this vastly increases the parameter space, and unfortunately we could not obtain good fits with simple analytical forms of the RKKY exchange. A more sophisticated approach, using the measured band structure of the Pd-U conduction band, may give better results. This consideration may also apply to the quadrupolar interaction parameters, and may account for the discrepancies between the measured and calculated phase boundaries.

Finally, we note that ultrasound measurements [35] and a symmetry analysis of the XRS data [36] showed that there is a sequence of structural transitions from hexagonal to orthorhombic symmetry at $T_{0}$ and from orthorhombic to monoclinic at $T_{+1}$. As the parameters in Table II have the symmetry of the high-temperature hexagonal structure, it is possible that using temperature dependent exchange parameters, which incorporate deviations from hexagonal symmetry proportional to the order parameter may yield a better fit.

The magnetoelastic strain is proportional to the strain derivative of the free energy [37],

$$
\epsilon^{\alpha}=-\sum_{\beta} \frac{s^{\alpha \beta}}{V} \frac{\partial F}{\partial \epsilon^{\beta}},
$$

where $s^{\alpha \beta}$ is the elastic compliance and the indices $\alpha$ and $\beta$ are the Cartesian directions. Noting that $F=-k_{B} T \ln Z$, where the partition function is $Z=\sum_{n} \exp \left(-E_{n} / k_{B} T\right)$, we thus find that $\frac{\partial F}{\partial \epsilon^{\beta}}=\left\langle\frac{\partial E_{n}}{\partial \epsilon^{\beta}}\right\rangle$ where the angled brackets indicate the thermal expectation value.

In the context of the Hamiltonian developed above, there are two main contributions to the magnetoelastic strain. These arise from the single-ion crystal field and the two-ion exchange interactions, both of which depend on the position of the ions, and are hence coupled to any change in the lattice. These two interactions give rise to crystal-field striction [38] and exchange striction [37], respectively. In order to calculate the magnetostriction, we thus have to find expressions for the energy levels $E_{n}$ as a function of these two interactions. This is done by expanding the Hamiltonian (4) in a Taylor series and making the harmonic approximation by keeping only the first-order term, which yields

$$
\begin{gathered}
\epsilon_{\mathrm{cf}}^{\alpha}=-\sum_{k q, i} \frac{s^{\alpha \beta}}{V} \frac{\partial B_{k}^{q}}{\partial \epsilon}\left\langle O_{k}^{q}\right\rangle, \\
\epsilon_{\mathrm{ex}}^{\alpha}=\frac{1}{2} \sum_{\beta, i j} \frac{s^{\alpha \beta}}{V} \frac{\partial \mathcal{J}_{i j}}{\partial \epsilon^{\beta}}\left\langle\hat{J}_{i}^{\beta} \hat{J}_{j}^{\beta}\right\rangle .
\end{gathered}
$$

The prefactors $A_{\alpha}=\frac{s^{\alpha \beta}}{V} \frac{\partial B_{q}^{k}}{\partial \epsilon}$ and $K_{\alpha \beta}^{i j}=\frac{s^{\alpha \beta}}{V} \frac{\partial \mathcal{J}_{i j}}{\partial \epsilon^{\beta}}$ may be taken to be independent of field and temperature and can then be fitted to experimental data given the thermal expectation 
TABLE III. Fitted magnetoelastic parameters.

\begin{tabular}{lrrr}
\hline \hline & \multicolumn{3}{c}{$\alpha$} \\
\cline { 2 - 4 } & \multicolumn{1}{c}{$a$} & \multicolumn{1}{c}{$b$} & $c$ \\
\hline$A_{\alpha, x y} \times 10^{-5}$ & 26 & -100 & \\
$A_{\alpha, y z} \times 10^{-5}$ & -35 & 85 & \\
$A_{\alpha, z^{2}} \times 10^{-5}$ & -1 & 5 & -30 \\
$A_{\alpha, z x} \times 10^{-5}$ & 80 & -50 & -30 \\
$A_{\alpha, x^{2}-y^{2}} \times 10^{-5}$ & 5 & -60 & -30 \\
$K_{\alpha, x y}^{a b} \times 10^{-5}$ & 48 & 20 & \\
$K_{\alpha, y z}^{a b} \times 10^{-5}$ & -10 & 30 & \\
$K_{\alpha, z x}^{a b} \times 10^{-5}$ & -150 & -45 & 25 \\
$K_{\alpha, x^{2}-y^{2}}^{a b} \times 10^{-5}$ & 1 & 10 & 10 \\
$K_{\alpha, x y}^{c} \times 10^{-5}$ & -48 & -14 & \\
$K_{\alpha, y z}^{c} \times 10^{-5}$ & -3 & -40 & 5 \\
$K_{\alpha, z x}^{c} \times 10^{-5}$ & 200 & 35 & \\
$K_{\alpha, x^{2}-y^{2}}^{c} \times 10^{-5}$ & 8 & -4 & \\
\hline \hline
\end{tabular}

values $\left\langle O_{k}^{q}\right\rangle$ and $\left\langle\hat{J}_{i} \hat{J}_{j}\right\rangle$ obtained from the mean-field model at different applied magnetic fields.

Considering only the nonzero terms up to rank 2 in the Hamiltonian (4), we thus have

$$
\begin{aligned}
\epsilon^{\alpha}= & \frac{1}{N} \sum_{i}\left[\sum_{\beta} A_{\alpha \beta}\left\langle\hat{Q}_{\beta}^{i}\right\rangle+\sum_{\beta} K_{\alpha \beta}^{a b}\left\langle\hat{Q}_{\beta}^{i} \hat{Q}_{\beta}^{i+(\mathbf{a}+\mathbf{b}) / 2}\right\rangle\right. \\
& \left.+\sum_{\beta} K_{\alpha \beta}^{c}\left\langle\hat{Q}_{\beta}^{i} \hat{Q}_{\beta}^{i+\mathbf{c}}\right\rangle\right],
\end{aligned}
$$

where $N=4$ is the number of $\mathrm{U}^{4+}$ ions in the magnetic unit cell, and the indices $\alpha=x, y, z, \beta=x y, y z, z^{2}, z x, x^{2}-y^{2}$. In order to reduce the number of parameters in fitting equation (10) to the data, we considered only the quadrupolar exchange interactions between nearest-neighbor ions in the $c$ direction and the basal plane, since the calculated expectation values of $\left\langle\hat{Q}_{i} \hat{Q}_{j}\right\rangle \gg\left\langle\hat{J}_{i} \hat{J}_{j}\right\rangle$. Table III shows the fitted parameters, whilst the calculated magnetostriction is shown as solid lines in Fig. 5.

The calculated magnetostriction fits the data well in the high-field regions, but does not reproduce the low-field behavior, particular for the case where the field is applied along $x$. It may be that at low fields, some of the measured magnetostriction is due to domain rotation, which is not considered in the mean-field model.

\section{CONCLUSIONS}

We have deduced a mean-field model including up to four nearest-neighbor dipolar exchange and quadrupolar interactions between the $5 f^{2}$ electrons of $\mathrm{UPd}_{3}$, which is in generally good qualitative agreement with a broad range of experimental results. The interactions between electrons on the quasicubic sites were deduced from resonant $\mathrm{x}$-ray scattering measurements of the quadrupolar order parameters of each of the four low-temperature ordered phases, and from the measured transition temperatures and critical fields. The model was then used to calculate the high-field magnetization and magnetostriction, and the magnetic phase diagram up to $30 \mathrm{~T}$. With an applied magnetic field in the basal plane, the calculations for the $x$ and $y$ directions agree with the data. The calculated magnetization with the field parallel to $z$ is a factor of 3 too large, however, and the calculated critical field in this direction is much smaller than that measured. Despite this, the $z$-direction calculated magnetic phase diagram qualitatively reproduces the measured one. In conclusion, we have developed a nearest-neighbor mean-field model that reproduces many of the main features observed in $\mathrm{UPd}_{3}$.

\section{ACKNOWLEDGMENTS}

The authors thank Jens Jensen, Gillian Gehring, Amanda Gipson, and Helen Walker for fruitful discussions. M.D.L. thanks the UK Engineering and Physical Sciences Research Council for a research studentship, and UCL Graduate School for a project grant. J.G.P. acknowledges assistance by G. Chouteau and the support provided by the Grenoble High Magnetic Field Laboratory. M.D.L. and M.R. acknowledge the support of the European Science Foundation's Cooperation in Science and Technology (COST) program, contract number COST-STSM-P16-02275. Experiments performed at the NHMFL were supported by NSF Cooperative Agreement DMR-0084173, by the state of Florida, and the US Department of Energy. Support by the Deustche Forschungsgemeinschaft (SFB463), and the Austrian FWF (P17226) is gratefully acknowledged.

\section{APPENDIX}

We derive in this appendix the form of the anisotropic exchange interactions used in Eq. (4). In addition to the isotropic terms of the form,

$$
\mathcal{H}_{\text {iso }}=\sum_{i j} \sum_{k} \mathcal{J}_{k}^{i j} \sum_{q} \hat{O}(i)_{k}^{q} \cdot \hat{O}(j)_{k}^{q},
$$

where the $\hat{O}(i)_{k}^{q}$ denote the Stevens operators of the $i$ th ion, we introduce local anisotropic couplings of the form

$$
\begin{aligned}
& \mathcal{H}_{\text {aniso }}^{\text {local }} \\
& =\sum_{i j} \sum_{k}\left\{\sum_{q=1}^{k} \mathcal{K}_{k q}^{\prime j j}\left[\hat{C}(i)_{k}^{q} \cdot \hat{C}(j)_{k}^{q}+\hat{C}(i)_{k}^{-q} \cdot \hat{C}(j)_{k}^{-q}\right]\right. \\
& \left.\quad+\mathcal{K}_{k 0}^{\prime i j} \hat{C}(i)_{k}^{0} \cdot \hat{C}(j)_{k}^{0}\right\},
\end{aligned}
$$

where the spherical tensor operators $\hat{C}_{k}^{q}$ (which are equivalent to spherical harmonics in a similar way to the equivalence of Stevens operators to tesseral harmonics) are defined as

$$
\begin{aligned}
\hat{C}_{k}^{-|q|} & =\frac{1}{\sqrt{2}}\left(\hat{O}_{k}^{|q|}-i \hat{O}_{k}^{-|q|}\right), \\
\hat{C}_{k}^{0} & =\hat{O}_{k}^{0}, \\
\hat{C}_{k}^{+|q|} & =\frac{(-1)^{|q|}}{\sqrt{2}}\left(\hat{O}_{k}^{|q|}+i \hat{O}_{k}^{-|q|}\right) .
\end{aligned}
$$


For the rank $k=1$ case, these are the familiar angular momentum ladder operators $\hat{C}_{1}^{1}=-\hat{J}_{+}, \hat{C}_{1}^{-1}=\hat{J}_{-}$, and $\hat{C}_{1}^{0}=\hat{J}_{z}$. In the same way, the rank $k=1$ Stevens operators are the same as the Cartesian angular momentum operators $\hat{O}_{1}^{1}=\hat{J}_{x}$, $\hat{O}_{1}^{-1}=\hat{J}_{y}$, and $\hat{O}_{1}^{0}=\hat{J}_{z}$. Thus the Cartesian form of Eq. (A2) for rank $k=1$ is

$$
\mathcal{H}_{\text {aniso }}^{1 \text { local }}=\sum_{i j} \mathcal{K}_{11}^{\prime i j}\left(\hat{J}_{x}^{i} \hat{J}_{x}^{j}-\hat{J}_{y}^{i} \hat{J}_{y}^{j}\right)+\mathcal{K}_{10}^{\prime i j} \hat{J}_{z}^{i} \hat{J}_{z}^{j}
$$

The above anisotropic Hamiltonian (A2) is expressed in the local frame defined by $x^{\prime}, y^{\prime}$, and $z^{\prime}$, where $x^{\prime}$ is perpendicular to the hexagonal $c$ axis, in the plane formed by the $c$ axis and the vector $\mathbf{r}_{i j}$ joining ions $i$ and $j ; y^{\prime}$ is perpendicular to $c$ and $x^{\prime}$ and $z^{\prime} \| c$. Thus to obtain the anisotropic couplings in the global coordinate system, one needs only to rotate Eq. (A2) by the angle $\phi_{i j}$ between the projection of $\mathbf{r}_{i j}$ into the $a b$ plane and the global $x$ axis (defined as parallel to hexagonal $a$ ).
We note that this form of the anisotropy explicitly assumes a planar character. A more general expression may be obtained by considering anisotropy along the $c$ direction, by using the local coordinate system defined by $z^{\prime} \| \mathbf{r}_{i j}$ [39], $y^{\prime}$ in the $a b$ plane and perpendicular to $z^{\prime}$ and $x^{\prime}$ perpendicular to both $y^{\prime}$ and $z^{\prime}$. In this case, two rotations, by $\theta_{i j}$ about $y^{\prime}$ to rotate $x^{\prime}$ into the $a b$ plane (and also making $z^{\prime} \| c$ ) and $\phi_{i j}$ to rotate $x^{\prime}$ to become parallel to $a$ are required to obtain the expressions in the global coordinate system.

The rank $k=1$ rotation matrix to accomplish this is [40]

$$
\mathbf{R}_{1}=\left(\begin{array}{ccc}
\frac{1}{2} e^{-i \phi}(1+\cos \theta) & \frac{e^{-i \phi} \sin \theta}{\sqrt{2}} & \frac{1}{2} e^{-i \phi}(1-\cos \theta) \\
-\frac{\sin \theta}{\sqrt{2}} & \cos \theta & \frac{\sin \theta}{\sqrt{2}} \\
\frac{1}{2} e^{i \phi}(1-\cos \theta) & \frac{e^{i \phi} \sin \theta}{\sqrt{2}} & \frac{1}{2} e^{i \phi}(1+\cos \theta)
\end{array}\right),
$$

where the subscripts $i j$ has been omitted for clarity, and the rank $k=2$ matrix is
$\mathbf{R}_{2}=\left(\begin{array}{c}\frac{1}{4} e^{-2 i \phi}(1+\cos \theta)^{2} \\ -\frac{1}{2} e^{-i \phi}(1+\cos \theta) \sin \theta \\ \frac{1}{2} \sqrt{\frac{3}{2}} \sin \theta^{2} \\ -\frac{1}{2} e^{i \phi}(1-\cos \theta) \sin \theta \\ \frac{1}{4} e^{2 i \phi}(1-\cos \theta)^{2}\end{array}\right.$

$$
\begin{gathered}
-\frac{1}{2} e^{-2 i \phi}(1+\cos \theta) \sin \theta \\
\frac{1}{2} e^{-i \phi}(1+\cos \theta)(-1+2 \cos \theta) \\
-\sqrt{6} \cos \theta \sin \theta \\
\frac{1}{2} e^{i \phi}(1-\cos \theta)(1+2 \cos \theta) \\
-\frac{1}{2} e^{2 i \phi}(1-\cos \theta) \sin \theta
\end{gathered}
$$

$$
\begin{gathered}
\frac{1}{2} e^{-2 i \phi}(1-\cos \theta) \sin \theta \\
\frac{1}{2} e^{-i \phi}(1-\cos \theta)(1+2 \cos \theta) \\
\sqrt{6} \cos \theta \sin \theta \\
\frac{1}{2} e^{i \phi}(1+\cos \theta)(-1+2 \cos \theta) \\
\frac{1}{2} e^{2 i \phi}(1+\cos \theta) \sin \theta
\end{gathered}
$$

Multiplying the tensor operators vector by this matrix then transforms the local anisotropic Hamiltonian into the global frame,

$$
\mathcal{H}_{\text {aniso }}^{\text {global }}=\sum_{i j} \sum_{k}\left[\mathbf{R}_{k}\left(\begin{array}{c}
\mathcal{K}_{k k}^{\prime i j} \hat{C}_{k}^{-k}(i) \\
\vdots \\
\mathcal{K}_{k k}^{\prime i j} \hat{C}_{k}^{k}(i)
\end{array}\right)\right] \cdot\left[\mathbf{R}_{k}\left(\begin{array}{c}
\hat{C}_{k}^{-k}(j) \\
\vdots \\
\hat{C}_{k}^{k}(j)
\end{array}\right)\right] .
$$

In the current case, we chose the local coordinates such that $\theta_{i j}=0$ so the matrices $R_{k}$ simplify to

$$
\mathbf{R}_{k}=\left(\begin{array}{ccc}
e^{-k i \phi} & & 0 \\
& \ddots & \\
0 & & e^{k i \phi}
\end{array}\right)
$$

and Eq. (A7) to

$$
\mathcal{H}_{\text {aniso }}^{\text {global }}=\sum_{i j} \sum_{k}\left\{\sum_{q=1}^{k} \mathcal{K}_{k q}^{\prime i j} e^{2 q i \phi_{i j}}\left[\hat{C}(i)_{k}^{q} \cdot \hat{C}(j)_{k}^{q}+\hat{C}(i)_{k}^{-q} \cdot \hat{C}(j)_{k}^{-q}\right]+\mathcal{K}_{k 0}^{\prime i j} \hat{C}(i)_{k}^{0} \cdot \hat{C}(j)_{k}^{0}\right\} .
$$

Using the definitions (A3), one may obtain the Hamiltonian (4), which in matrix notation is

$$
\begin{aligned}
\mathcal{H}_{k} & =\sum_{i j} \mathbf{J}_{k}^{\ddot{j}} \mathbf{O}(i) \otimes \mathbf{O}(j), \\
\mathbf{O}(i) & =\left(\begin{array}{c}
\hat{O}_{k}^{-k}(i) \\
\vdots \\
\hat{O}_{k}^{k}(i)
\end{array}\right),
\end{aligned}
$$


where the exchange coupling matrices are, for rank $k=1$,

$$
\mathbf{J}_{1}^{i j}=\left(\begin{array}{ccc}
\mathcal{J}_{11}^{i j}-\mathcal{K}^{+i j} & 0 & -\mathcal{K}^{-i j}{ }_{11} \\
0 & \mathcal{J}_{10}^{i j} & 0 \\
-\mathcal{K}^{-i j} & 0 & \mathcal{J}_{11}^{i j}+\mathcal{K}^{+i j}{ }_{11}
\end{array}\right),
$$

where $\mathcal{K}^{ \pm}{ }_{k q}^{\ddot{j}}$ denote $\mathcal{K}_{k q}^{i j} \cos q \phi_{i j}$ and $\mathcal{K}_{k q}^{i j} \sin q \phi_{i j}$, respectively, and the rank $k=2$ matrices are

$$
\mathbf{J}_{2}^{i j}=\left(\begin{array}{ccccc}
\mathcal{J}_{22}^{i j}-\mathcal{K}^{+i j} & 0 & 0 & 0 & -\mathcal{K}^{-i j} \\
0 & \mathcal{J}_{21}^{i j}-\mathcal{K}^{+i j} & 0 & -\mathcal{K}^{-i j} & 0 \\
0 & 0 & 0 & 0 & 0 \\
0 & -\mathcal{K}^{-i j} & 0 & \mathcal{J}_{21}^{i j}+\mathcal{K}^{+i j} & 0 \\
-\mathcal{K}_{21}^{-i j} & 0 & 0 & 0 & \mathcal{J}_{22}^{i j}+\mathcal{K}^{+i j}{ }_{22}
\end{array}\right)=\left(\begin{array}{ccccc}
\mathcal{J}_{x y}^{i j} & 0 & 0 & 0 & -\mathcal{K}^{-i j} \\
0 & \mathcal{J}_{y z}^{i j} & 0 & -\mathcal{K}^{-i j} & 0 \\
0 & 0 & 0 & 0 & 0 \\
0 & -\mathcal{K}^{-i j} & 0 & \mathcal{J}_{z x}^{i j} & 0 \\
-\mathcal{K}^{-i j} & 0 & 0 & 0 & \mathcal{J}_{x^{2}-y^{2}}^{i j}
\end{array}\right) .
$$

The restrictions (5) and (6) imply that $\mathcal{J}_{z x}^{\text {nnc }}<0, \mathcal{J}_{x y}^{\text {nnc }}>0,\left|\mathcal{J}_{z x}^{\text {nnc }}\right|>\left|\mathcal{J}_{x y}^{\text {nnc }}\right|$, and $\mathcal{J}_{z x}^{\text {nn }}<\mathcal{J}_{x y}^{\text {nnc }}<0$.

[1] P. Santini, S. Carretta, G. Amoretti, R. Caciuffo, N. Magnani, and G. H. Lander, Rev. Mod. Phys. 81, 807 (2009).

[2] F. Yakhou, V. Plakhty, H. Suzuki, S. Gavrilov, P. Burlet, L. Paolasini, C. Vettier, and S. Kunii, Phys. Lett. A 285, 191 (2001).

[3] H. Nakao, K. Magishi, Y. Wakabayashi, Y. Murakami, K. Koyama, K. Hirota, Y. Endoh, and S. Kunii, J. Phys. Soc. Jpn. 70, 1857 (2001).

[4] T. Onimaru, T. Sakakibara, T. Tayama, N. Aso, H. Y. amd Dai Aoki, Y. Ōnuki, T. Kawae, T. Kitai, and T. Takeuchi, Physica B 359-361, 935 (2005).

[5] T. Nagao and R. Shiina, J. Phys. Soc. Jpn. 79, 034703 (2010).

[6] Y. Tanaka, T. Inami, S. W. Lovesey,, K. S. Knight, F. Yakhou, D. Mannix, J. Kokubun, M. Kanazawa, K. Ishida, S. Nanao, T. Nakamura, H. Yamauchi, H. Onodera, K. Ohoyama, and Y. Yamaguchi, Phys. Rev. B 69, 024417 (2004).

[7] S. W. Zochowski, M. de Podesta, C. Lester, and K. A. McEwen, Physica B 206\&207, 489 (1995).

[8] K. A. McEwen, J.-G. Park, A. J. Gipson, and G. A. Gehring, J. Phys.: Condens. Matter 15, S1923 (2003).

[9] Y. Tokiwa, K. Sugiyama, T. Takeuchi, M. Nakashima, R. Settai, Y. Inada, Y. Haga, E. Yamamoto, K. Kindo, H. Harima, and Y. Onuki, J. Phys. Soc. Jpn. 70, 1731 (2001).

[10] U. Steigenberger, K. A. McEwen, J. L. Martinez, and D. Fort, J. Magn. Magn. Mater. 108, 163 (1992).

[11] D. F. McMorrow, K. A. McEwen, U. Steigenberger, H. M. Rønnow, and F. Yakhou, Phys. Rev. Lett. 87, 057201 (2001).

[12] H. C. Walker, K. A. McEwen, D. F. McMorrow, S. B. Wilkins, F. Wastin, E. Colineau, and D. Fort, Phys. Rev. Lett. 97, 137203 (2006).

[13] H. C. Walker, K. A. McEwen, M. D. Le, L. Paolasini, and D. Fort, J. Phys.: Condens. Matter 20, 395221 (2008).

[14] T. J. Heal and G. I. Williams, Acta. Cryst. 8, 494 (1955).

[15] H. H. Hill, Nucl. Metall. 17, 2 (1970).

[16] K. T. Moore and G. van der Laan, Rev. Mod. Phys. 81, 235 (2009).

[17] K. A. McEwen, H. C. Walker, M. D. Le, D. F. McMorrow, E. Colineau, F. Wastin, S. B. Wilkins, J.-G. Park, R. I. Bewley, and D. Fort, J. Magn. Magn. Mater. 310, 718 (2007).
[18] A. Martin-Martin, Ph.D. thesis, University College London, 2000.

[19] M. D. Le, K. A. McEwen, M. Rotter, J. Jensen, R. I. Bewley, T. Guidi, and D. Fort, J. Phys.: Condens. Matter 24, 036002 (2012).

[20] D. J. Newman and B. K. C. Ng, Crystal Field Handbook (Cambridge University Press, Cambridge, UK, 2000).

[21] G. A. Gehring (private communication).

[22] W. J. L. Buyers, A. F. Murray, T. M. Holden, E. C. Svensson, P. de V. DuPlessis, G. H. Lander, and O. Vogt, Physica B+C 102, 291 (1980).

[23] K. R. Lea, M. J. M. Leask, and W. P. Wolf, J. Phys. Chem. Solids 23, 1381 (1962).

[24] K. A. McEwen, U. Steigenberger, K. N. Clausen, J. Kulda, J.-G. Park, and M. B. Walker, J. Magn. Magn. Mater. 177-181, 37 (1998).

[25] M. Rotter, H. Müller, E. Gratz, M. Doerr, and M. Loewenhaupt, Rev. Sci. Instr. 69, 2742 (1998).

[26] S. W. Zochowski and K. A. McEwen, Physica B 199\&200, 416 (1994).

[27] M. Rotter, J. Magn. Magn. Mater. 272-276, E481 (2004).

[28] M. Rotter et al., http://www.mcphase.de

[29] R. A. Cowley and J. Jensen, J. Phys.: Condens. Matter 4, 9673 (1992).

[30] J. G. Houmann, B. D. Rainford, J. Jensen, and A. R. Mackintosh, Phys. Rev. B 20, 1105 (1979).

[31] J. Jensen and A. R. Mackintosh, Rare Earth Magnetism (Clarendon Press, Oxford, 1991).

[32] For a two-level system, the mean-field susceptibility is inversely proportional to $\Delta\left(\Delta-2 n_{s d}|\langle d|\hat{O}| s\rangle|^{2} \mathcal{J}(\mathbf{q})\right)$, which may be equated with the Curie-Weiss susceptibility, $\chi \propto 1 /\left(T-T_{N}\right)$. At low temperatures, $T \ll \Delta$, the thermal population factor $n_{s d} \approx \Delta / k_{B} T$, so that $T_{N} \stackrel{\alpha}{\sim}|\langle d|\hat{O}| s\rangle|^{2} \mathcal{J}(\mathbf{q})$.

[33] J. Kennedy and R. Eberhart, in Proceedings of IEEE International Conference on Neural Networks, Vol. 4 (IEEE, Piscataway, NJ, 1995), pp. 1942-1948.

[34] K. A. McEwen, U. Steigenberger, and J. L. Martinez, Physica B 186-188, 670 (1993). 
[35] N. Lingg, D. Maurer, V. Müller, and K. A. McEwen, Phys. Rev. B 60, R8430 (1999).

[36] J. Fernández-Rodríguez, S. W. Lovesey, and J. A. Blanco, J. Phys.: Condens. Matter 22, 022202 (2010).

[37] M. Doerr, M. Rotter, and A. Lindbaum, Adv. Phys. 54, 1 (2005).
[38] P. Morin and D. Schmitt, in Ferromagnetic Materials, edited by K. H. J. Buschow and E. P. Wohlfarth, Vol. 5 (Elsevier, Amsterdam, 1990), pp. 1-132.

[39] J. Jensen (private communication).

[40] H. A. Buckmaster, R. Chatterjee, and Y. H. Shing, Phys. Stat. Sol. (a) 13, 9 (1972). 